\title{
Effect of the types of stabilizers and size distribution on catalytic activity of palladium nanoparticles in the carboxylative coupling reaction
}

\author{
Jian Sun ${ }^{1} \cdot$ Dailin $\mathrm{Li}^{1} \cdot$ Huajing Gao ${ }^{1}$ Zhaohui Jin ${ }^{1} \cdot$ Ming Bao $^{2}$
}

(c) Springer Nature Switzerland AG 2018

\begin{abstract}
Palladium nanoparticles (Pd NPs) generated in situ and stabilized by quaternary ammonium salts were used in threecomponent coupling reaction as the catalyst. Cations and anions of quaternary ammonium salts influenced the catalytic activity of Pd NPs, and tetrabutyl-ammonium bromide (TBAB) proved to be the best stabilizer. $(2.23 \pm 0.58) \mathrm{nm}$-sized Pd NPs showed the best catalytic activity under the molar ratio $1 / 30\left[\mathrm{Pd}(\mathrm{acac})_{2} / \mathrm{TBAB}\right]$. The mercury poisoning test demonstrated the reaction occurred on the surface of Pd NPs. Pd NPs could be recycled for four times without significant loss in catalytic activity.
\end{abstract}

Graphical abstract The mercury poisoning tests demonstrated carboxylative coupling reaction occurred on the surface of Pd NPs which generated in situ, the types and additive amount of stabilizers influenced the catalytic activity of this catalytic system which could be recycled.

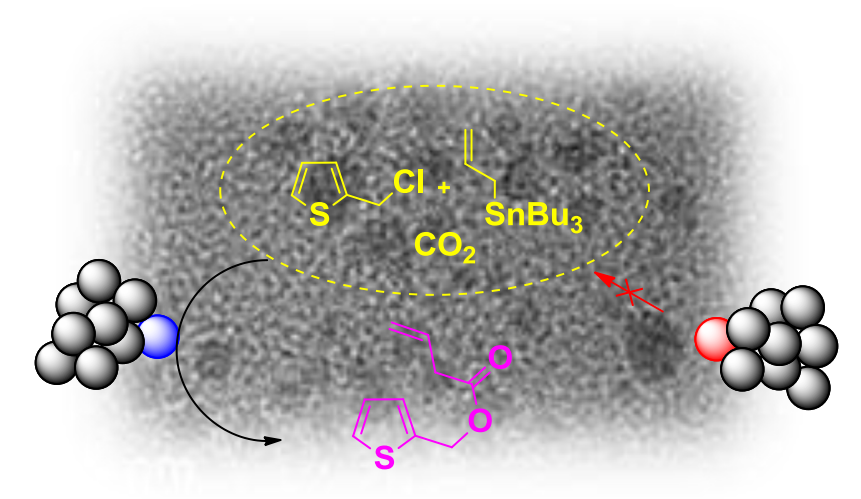

Keywords Stabilizer $\cdot$ Size distribution $\cdot$ Nanoparticles $\cdot$ Carboxylative coupling $\cdot \mathrm{CO}_{2} \cdot$ Organic

\footnotetext{
$\triangle$ Jian Sun, sunjian6225@126.com; $\bowtie$ Huajing Gao, huajing_gao@126.com | 'Institute of Petrochemical Technology, Jilin Institute of Chemical Technology, Jilin 132022, China. ${ }^{2}$ State Key Laboratory of Fine Chemicals, Dalian University of Technology, Dalian 116023 , China.
} 


\section{Introduction}

Over the past few years, metal nanoparticles (MNPs) catalysts have attracted much interest and been widely used in many types of coupling reactions [1-3], such as Heck [4], Suzuki [5, 6], Sonogashira [7, 8], Stille [9, 10], Ullmann $[11,12]$, and Hiyama reactions $[13,14]$. MNPs catalysts are usually better than the corresponding molecular catalysts on catalytic activity due to their large surface area, so, they need protective agents to avoid the formation of bulk metal. MNPs are generally stabilised by polymeric molecules $[15,16]$, tetraalkylammonium salts $[17,18]$, and ionic liquids $[19,20]$. The first example of Heck coupling reactions under ligand-free conditions was successfully conducted by using $\mathrm{Pd}(\mathrm{OAc})_{2} /{ }^{n} \mathrm{Bu}_{4} \mathrm{NCl}$ as the catalyst and phase-transfer catalyst in 1984, however, no person realized that this catalytic system was promoted by Pd NPs at that time [21]. Recently, the carboxylative coupling reactions were successfully conducted by our group using $\mathrm{Pd}$ NPs in the presence of quaternary ammonium salts as the stabilizer, but the effect of quaternary ammonium salts and size distribution of Pd NPs on catalytic activity was not discussed [22].

Herein, we use the carboxylative coupling reaction of (2-chloromethyl)thiophene, allyltributylstannane, and carbon dioxide as the model reaction (Scheme 1), reveal the effect of the types of stabilizers and size distribution on catalytic activity of Pd NPs by transmission electron microscopy (TEM) studies, confirm whether the reaction occurred on the surface of Pd NPs, and explore the recyclability of this catalytic system.

\section{Experimental}

\subsection{Catalytic activity measurement of Pd NPs stabilized by different types of quaternary ammonium salts}

$\mathrm{Pd}(\mathrm{acac})_{2}(7.6 \mathrm{mg}, 0.025 \mathrm{mmol})$, quaternary ammonium salt $(0.7 \mathrm{mmol}), 2$-(chloromethyl)-thiophene $(66.0 \mathrm{mg}$, $0.5 \mathrm{mmol})$, allyltributylstannane (199.0 $\mathrm{mg}, 0.6 \mathrm{mmol})$, and THF $(5 \mathrm{~mL})$ were placed in a $50 \mathrm{~mL}$ autoclave with a magnetic stir bar under a $\mathrm{N}_{2}$ atmosphere. The autoclave was purged with $\mathrm{CO}_{2}$ three times, filled with $\mathrm{CO}_{2}$ to $2 \mathrm{MPa}$ pressure, and heated to $70^{\circ} \mathrm{C}$ for $24 \mathrm{~h}$. The autoclave was allowed to cool to room temperature and the remaining $\mathrm{CO}_{2}$ was vented. The resultant mixture was evaporated in vacuo to give the crude product, which was then purified via silica gel chromatography (eluent: ethyl acetate/petroleum ether $=1: 20$ ) to afford benzyl but-3-enoate as a yellowish oil [22]. (TMAB: tetramethyl-ammonium bromide; TOAB: tetraoctyl-ammonium bromide; CTMAB: hexadecyl trimethyl ammonium bromide; TBAF: tetrabutyl-ammonium fluoride; TBAC: tetrabutyl-ammonium chloride; TBAl: tetrabutyl-ammonium iodide.) TEM was performed on a Tecnai G2 microscope operating at $120 \mathrm{kV}$. After the carboxylative coupling reaction of 2-(chloromethyl)-thiophene with allyltributylstannane was performed for $24 \mathrm{~h}$ under optimized reaction conditions, the resultant mixture was used for TEM determination.

\subsection{Size distribution control and catalytic activity measurement of Pd NPs by variation of amount of TBAB}

In the case of this reaction, we used TBAB in different molar ratio with respect to $\mathrm{Pd}(\mathrm{acac})_{2}$ like 10:1, 20:1, 30:1, and 40:1 to see if it affected the size of Pd NPs, the reaction was conducted using the same procedure described above. The gradual variation in size of nanoparticles with different molar ratio is revealed clearly in the TEM studies.

\section{$2.3 \mathrm{Hg}(0)$ poisoning test}

As above procedure, a reaction of 2-(chloromethyl)thiophene $(66.0 \mathrm{mg}, 0.5 \mathrm{mmol})$, allyltributylstannane (199.0 mg, $0.6 \mathrm{mmol}), \mathrm{Pd}(\mathrm{acac})_{2}(7.6 \mathrm{mg}, 0.025 \mathrm{mmol})$, quaternary ammonium salt $(0.7 \mathrm{mmol})$, THF $(5 \mathrm{~mL})$, with the addition of elemental mercury $(2.5 \mathrm{mmol}, 100$ equiv, $501.5 \mathrm{mg}$ ) (relative to palladium), and were placed in a $50 \mathrm{~mL}$ autoclave with a magnetic stir bar under a $\mathrm{N}_{2}$ atmosphere. The autoclave was purged with $\mathrm{CO}_{2}$ three times, filled with $\mathrm{CO}_{2}$ to $2 \mathrm{MPa}$ pressure, and heated to $70{ }^{\circ} \mathrm{C}$ for $24 \mathrm{~h}[23]$.

\subsection{Recycling experiment}

After reaction completion, the mixture was then extracted with $n$-hexane $(3 \times 5 \mathrm{~mL})$. The top layer was collected and purified via silica gel chromatography. The Pd NPs (under
Scheme 1 Pd NPs-catalyzed carboxylative coupling reaction

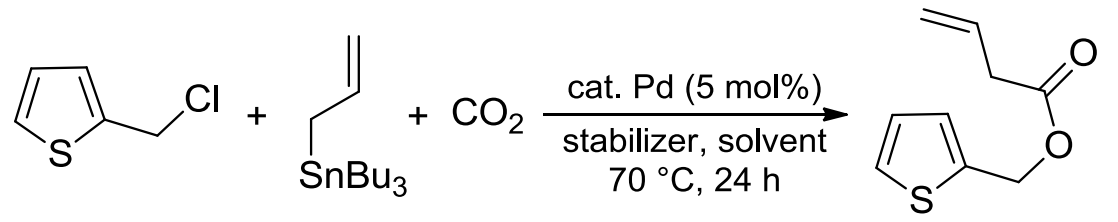


layer) was subjected to another run by charging it with the same substrates as mentioned above without further addition of $\mathrm{Pd}(\mathrm{acac})_{2}$ and TBAB.

\section{Result and discussion}

The TEM studies of Pd NPs by using quaternary ammonium salts as the stabilizer with different cations are shown in Fig. 1. It is seen that the serious conglobation when used TMAB or CTMAB as the stabilizer, and the yield of the product is 0 or $20 \%$, respectively. On the other hand, the uniform distribution and good dispersibility of Pd NPs are observed when the TBAB added with the $73 \%$ yield of the product. Although the dispersed nanoparticles are seen when TOAB as the stabilizer, conglobation can not be prevented with the $57 \%$ yield of the product. The results indicated that the quaternary ammonium salts with longer or shorter alkyl chains in cations are not conducive to the dispersion of Pd NPs, and higher catalytic activity is revealed when $T B A B$ is added which including ${ }^{n} \mathrm{Bu}_{4} \mathrm{~N}^{+}$.

Figure 2 shows the TEM studies of Pd NPs by using quaternary ammonium salts as the stabilizer with different anions. The Pd NPs are serious coated by TBAF, TBAC or TBAI, this phenomenon leads to the decrease of its catalytic activity, and the yield of the product is $13 \%, 25 \%$ or $17 \%$, respectively. Better catalytic activity and uniform dispersion are observed when using TBAB as the stabilizer.

The effect of stabilizer on the catalytic activity of Pd NPs, not only because of the types of stabilizers, but also due to the size of Pd NPs. TEM studies of Pd NPs with varying $\mathrm{Pd} / \mathrm{TBAB}$ molar ratio and their corresponding size distribution images were shown in Fig. 3. Size distribution of Pd NPs were $3.56 \pm 0.77 \mathrm{~nm}$ and $3.37 \pm 0.60 \mathrm{~nm}$ when $\mathrm{Pd} / \mathrm{TBAB}$ molar ratio $1 / 10$ and $1 / 20$ were used. At this moment, larger particle size and lower catalytic activity were observed with the $7 \%$ and $41 \%$ yields of the product, and serious conglobation were revealed. Pd NPs with more average and smaller diameter of $2.23 \pm 0.58 \mathrm{~nm}$ were generated when $\mathrm{Pd} / \mathrm{TBAB}$ molar ratio $1 / 30$ was used, the best catalytic activity and yield (73\%) were achieved in this case. However, continued to increase the molar ratio to $1 / 40$, no significant loss in catalytic activity ( $70 \%$ yield) with the diameter of $2.50 \pm 0.48 \mathrm{~nm}$.

Based on the TEM studies, a plausible growth mechanism for Pd NPs was speculated. Pd-rich and Pd-poor phases were formed with the increase of $\mathrm{Pd} / \mathrm{TBAB}$ molar ratio, then nucleation of amorphous nanoclusters within the Pd-rich phase, followed by crystallization of these amorphous clusters to formed Pd NPs with an ionic coating [24]. Although the Pd NPs showed good catalytic activity which generated in situ, the catalysis which occurred on the Pd NPs surface or by leached Pd species was still needed to assess. A large excess of $\mathrm{Hg}(0)$ was added to the model reaction under the standard conditions and no product was observed when the reaction finished. The $\mathrm{Hg}(0)$ poisoning experiments suggested that the reaction occurred on the Pd NPs surface and the active catalyst was very likely to be heterogeneous in nature. From the above, the nature of this catalytic system was not like a

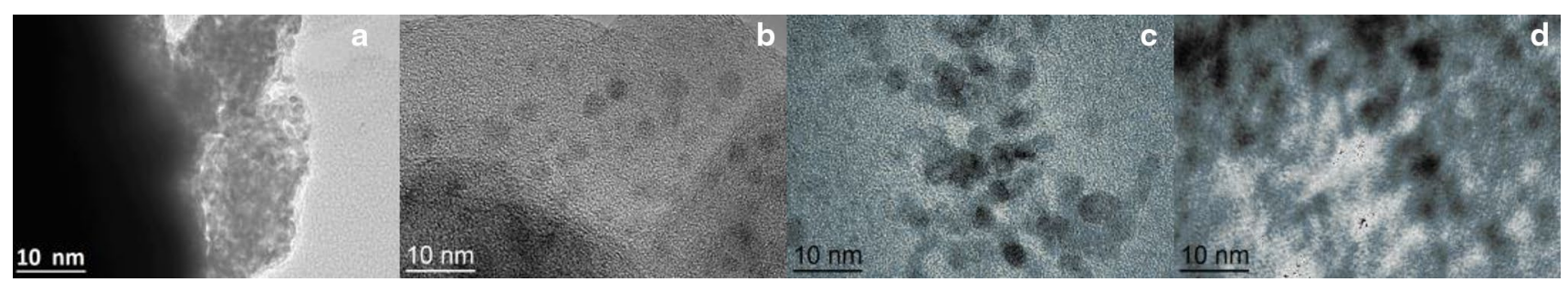

Fig. 1 TEM studies of palladium nanoparticles by using quaternary ammonium salts as the stabilizer with different cations: a TMAB, $\mathbf{b}$ TBAB, c TOAB, and d CTMAB

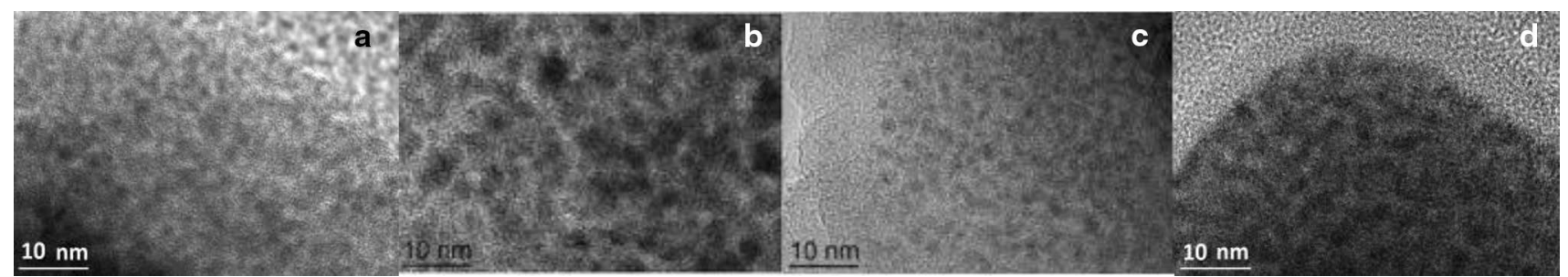

Fig. 2 TEM studies of palladium nanoparticles stabilized by quaternary ammonium salts containing different anions: a TBAF, b TBAC, c TBAB, d TBAI 

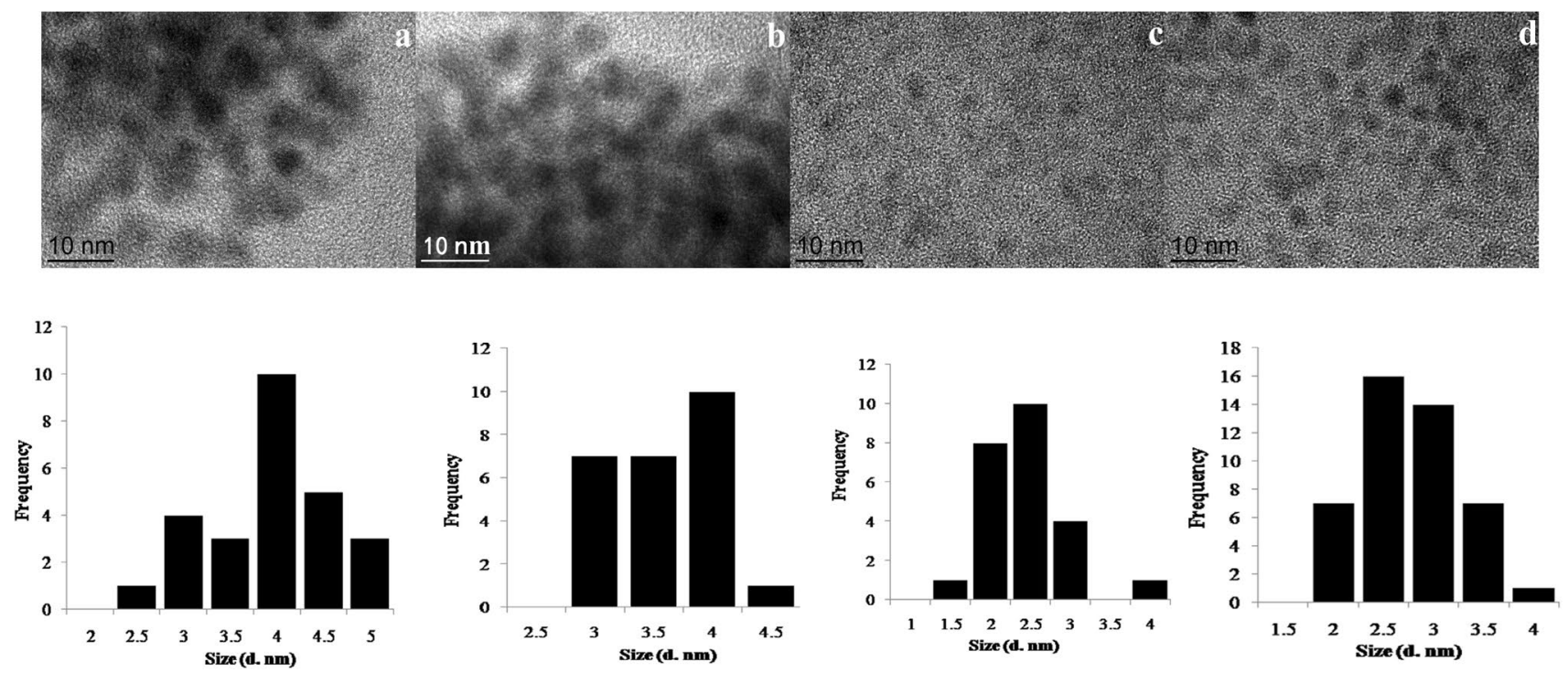

Fig. 3 TEM studies (up) of palladium nanoparticles with varying Pd/TBAB molar ratio and their corresponding size distribution images (down): a $1 / 10$, b $1 / 20$, c $1 / 30$, d $1 / 40$

Cocktail-type system, because no leaching for Pd and only Pd NPs were formed with an ionic coating in the catalytic system [25].

Studies were also conducted to assess the potential for recycling of the reaction medium. The trend of aggregation was observed after the two run recycling uses of Pd NPs, but without significant loss in catalytic activity. Pd black was found after four running four times, which leaded to a loss of the catalytic activity and the yield of product from $73 \%$ decrease to $54 \%$.

\section{Conclusion}

In summary, we have successfully illustrated that the effects of types of stabilizers and size distribution of $\mathrm{Pd}$ NPs on the catalytic activity in the carboxylative coupling reaction of (2-chloromethyl)thiophene, allyltributylstannane, and carbon dioxide. Excellent catalytic activity was observed using TBAB as the stabilizer with an average and smaller diameter of $2.23 \pm 0.58 \mathrm{~nm}$ under Pd/TBAB molar ratio $1 / 30$. The $\mathrm{Hg}(0)$ poisoning experiment suggested that the active catalyst was very likely to be heterogeneous in nature. Notably, this catalytic system generated in situ and could be recyclable. Further studies focusing on the reactions using magnetic Pd NPs as the catalyst are currently ongoing.

Acknowledgements This work was supported by the National Natural Science Foundation of China (Nos. 21372035, 21373041 and 21773021), and the Doctor Startup Foundation of Jilin Institute of Chemical Technology.

\section{Compliance with ethical standards}

Conflict of interest The authors declare that they have no conflict of interest.

\section{References}

1. Balanta A, Godard C, Claver C (2011) Pd nanoparticles for C-C coupling reactions. Chem Soc Rev 40:4973-4985

2. Astruc $D$ (2008) Nanoparticles and catalysis. Wiley, Weinheim

3. Astruc D, Lu F, Aranzaes J (2005) Nanoparticles as recyclable catalysts: the frontier between homogeneous and heterogeneous catalysis. Angew Chem Int Ed 44:7852-7872

4. Reetz M, Vries J (2004) Ligand-free heck reactions using low Pdloading. Chem Commun 40:1559-1563

5. Zhou Q, Wei S, Han W (2014) In situ generation of Palladium nanoparticles: ligand-free Palladium catalyzed pivalic acid assisted carbonylative Suzuki reactions at ambient conditions. J Org Chem 79:1454-1460

6. Das P, Sharma D, Shil A, Kumari A (2011) Solid-supported palladium nano and microparticles: an efficient heterogeneous catalyst for ligand-free Suzuki-Miyaura cross coupling reaction. Tetrahedron Lett 52:1176-1178

7. Rumi L, Scheuermann G, Mülhaupt R, Bannwarth W (2011) Palladium nanoparticles on graphite oxide as catalyst for SuzukiMiyaura, Mizoroki-Heck, and Sonogashira reactions. Helv Chim Acta 94:966-976

8. Gao S, Zhao N, Shu M, Che S (2010) Palladium nanoparticles supported on MOF-5: a highly active catalyst for a ligand- and copper-free Sonogashira coupling reaction. Appl Catal A Gen 388:196-201

9. Nasrollahzadeh M, Sajadi S, Honarmand E, Maham M (2015) Preparation of palladium nanoparticles using Euphorbia thymifolia L. leaf extract and evaluation of catalytic activity in the ligand-free Stille and Hiyama cross-coupling reactions in water. New J Chem 39:4745-4752 
10. Briggs B, Bedford N, Seifert S, Koerner H, Dakhel H, Heinz $H$, Naik R, Frenkel A, Knecht M (2015) Atomic-scale identification of Pd leaching in nanoparticle catalyzed $\mathrm{C}-\mathrm{C}$ coupling: effects of particle surface disorder. Chem Sci 6:6413-6419

11. Crabbe B, Kuehm O, Bennett J, Tapley G (2018) Light-activated Ullmann homocoupling of aryl halides catalyzed using gold nanoparticle-functionalized potassium Niobium oxides. Catal Sci Technol 8:4907-4915

12. Nejati K, Ahmadi S, Nikpassand M, Nezhad P, Vessally E (2018) Diaryl ethers synthesis: nano-catalysts in carbon-oxygen crosscoupling reactions. RSC Adv 8:19125-19143

13. Song J, Feng $X$, Yamamoto $Y$, Almansour Al, Arumugam N, Kumar RS, Bao M (2017) Carboxylative coupling of chloromethyl(hetero)arenes with allyl-trimethoxysilane catalyzed by Palladium nanoparticles. Asian J Org Chem 6:177-183

14. Chatterjee T, Dey R, Ranu B (2011) An easy access to styrenes: trans aryl 1,3-, 1,4- and 1,5-dienes, and 1,3,5-trienes by Hiyama cross-coupling catalyzed by palladium nanoparticles. New J Chem 35:1103-1110

15. Hattemer G, Arya G (2015) Viscoelastic properties of polymergrafted nanoparticle composites from molecular dynamics simulations. Macromolecules 48:1240-1255

16. Zhang J, Wei G, Keller T, Gallagher H, Stötzel C, Müller F, Gottschaldt M, Schubert U, Jandt K (2010) Responsive hybrid polymeric/metallic nanoparticles for catalytic applications. Macromol Mater Eng 295:1049-1057

17. Sun J, Wang J, Feng $X$, Yamamoto $Y$, Almansour Al, Arumugam N, Kumar RS, Bao M (2018) Carboxylative Suzuki coupling reactions of benzyl chlorides with allyl pinacolborate catalyzed by palladium nanoparticles. Chin J Catal 39:1258-1262

18. Sun J, Feng X, Zhao Z, Yamamoto Y, Bao M (2014) Carbonylative Stille coupling reactions of benzyl chlorides with allyltributylstannane catalyzed by palladium nanoparticles. Tetrahedron 70:7166-7171

19. Uematsu T, Baba M, Oshima Y, Tsuda T, Torimoto T, Kuwabata $S$ (2014) Atomic resolution imaging of gold nanoparticle generation and growth in ionic liquids. J Am Chem Soc 136:13789-13797

20. Schadt K, Kerscher B, Thomann R, Mülhaupt R (2013) Structured semifluorinated polymer ionic liquids for metal nanoparticle preparation and dispersion in fluorous compartments. Macromolecules 46:4799-4804

21. Jeffery T (1984) Palladium-catalysed vinylation of organic halides under solid-liquid phase transfer conditions. J Chem Soc Chem Commun 19:1287-1289

22. Sun J, Bao M, Feng X, Yu X, Yamamoto Y, Almansour Al, Arumugam N, Kumar RS (2015) Carboxylative coupling reaction of five-membered (chloromethyl) heteroarenes with allyltributylstannane catalyzed by palladium nanoparticles. Tetrahedron Lett 56:6747-6750

23. Widegren J, Finke R (2003) A review of the problem of distinguishing true homogeneous catalysis from soluble or other metal-particle heterogeneous catalysis under reducing conditions. J Mol Catal A Chem 198:317-341

24. Loh ND, Sen S, Bosman M, Tan SF, Zhong J, Nijhuis CA, Král $P$, Matsudaira P, Mirsaidov U (2017) Multistep nucleation of nanocrystals in aqueous solution. Nat Chem 9:77-82

25. Eremin DB, Ananikov VP (2017) Understanding active species in catalytic transformations: from molecular catalysis to nanoparticles, leaching, "Cocktails" of catalysts and dynamic systems. Coordin Chem Rev 346:2-19 